\title{
On QoS Mapping in TDMA Based Wireless Sensor Networks
}

\author{
Wassim Masri and Zoubir Mammeri \\ IRIT - Paul Sabatier University \\ Toulouse, France \\ \{Wassim.Masri, Zoubir.Mammeri\}@ @irit.fr
}

\begin{abstract}
Recently, there have been many efforts to develop Wireless Sensor Networks (WSN), but no one can deny the fact that QoS support is still one of the least explored areas in this domain. In this paper, we present QoS support in WSN while highlighting the QoS mapping issue, a complex process in which QoS parameters are translated from level to level and we present a case study of a TDMA tree-based clustered WSN, where accuracy and density on the user level are mapped to bandwidth on the network level. We end our paper with simulations that prove our formulas and highlight the relationships between QoS parameters.
\end{abstract}

\section{Introduction}

Wireless sensor networks (WSN) were named recently one of eight technologies to save the world [1], side-by-side with nuclear waste neutralizers, and one of ten emerging technologies that will change the world [2]. WSN are being integrated more and more in real world applications, they are used in the medical and healthcare domain, in the musical industry, and in a lot more real world applications.

While there have been many efforts to develop many facets in WSN, including hardware devices, communication protocols, energy consumption [3], and many other issues like time synchronization, geographical location and security, the QoS support remains one of the least explored areas in the WSN domain [4], since most of the research industry is following the actual trend in focusing on the energy consumption problem and the related issues.

In the matter of fact, QoS support in WSN could be seen from different points of view due to the layered architecture of typical distributed systems, so it could be seen as accuracy, density, precision or lifetime from the user's point of view, as well as bandwidth, delay or jitter from the network's point of view. Due to this 
diversity in seeing QoS, it is crucial to find the relationships between those different QoS requirements on the different levels, in order to obtain a coherent system. Once the relationships between those different parameters are found, one could translate them correctly from level to level, a process known as QoS mapping. QoS mapping is one of the least surveyed issues in the QoS management family, due to its complexity and its application dependency.

In this paper we intend to show the relationships between the density of a WSN (a user level QoS) and the bandwidth reserved for each of its nodes (a network level QoS). We prove the tight coupling between those two parameters on a TDMA tree based clustered WSN, while giving the length of a TDMA superframe as a function of the tree depth. We explore also the different scenarios while increasing the problem complexity. In order to validate our theoretical results, we present simulations that further highlight the relationships between the previously discussed QoS parameters.

In section 2, we present some related work to QoS support in WSN and QoS mapping. In section 3, we present our work on QoS mapping in WSN, and we show our novel approach on how we map user level QoS parameters: density and accuracy, to a network level QoS parameter, the bandwidth. This is achieved by computing the TDMA superframe length in several cases as a function of the network density. In section 4 , we present our simulations that validate our formulas presented in section 3, and we discuss the relationships between accuracy, density, reporting period, packet size and bandwidth. As far as we know, no one had explored the relationships between those parameters ever before. We end our paper in section 5 with some perspectives and conclusion.

\section{Related Work}

QoS support has a wide meaning, because it has not one common definition, beside, it could be seen on different levels and from different points of view.

In [5], QoS was defined to mean sensor network resolution. More specifically they defined it as the optimum number of sensors sending information toward information-collecting sinks, typically base stations. In [6], the authors used the Gur Game paradigm based on localized information to control the number of nodes to power up in a certain area, thus defining their QoS requirements as the optimal number of active nodes in the network. In [7], QoS was defined as the desired number of active sensors in the network, and the network lifetime as the duration for which the desired QoS is maintained.

We noticed that there are limited research papers about QoS mapping, because it is complex and application specific. For the best of our knowledge, very few works were done concerning QoS mapping in WSN. In [8], the authors presented a formal methodology to map application level SLA (response time) to network performance (link bandwidth and router throughput). In [9], the authors proposed 
a framework to ease the mapping between different Internet domains, namely between Intserv/RSVP and Diffserv domains. In [10], the authors proposed a framework to map the network packet loss rate to user packet loss rate by determining the location of each lost packet on the packet stream and calculating the impact of the lost packets on the application layer frames sent by a source. In WSN domain on the other hand, the work done concerning QoS mapping is so limited, and it covers mostly the tradeoffs between QoS parameters like energy, accuracy, density, latency, etc. In [11], simulations on QoS parameters in WSN were done, which led to a better understanding of the tradeoffs between density, latency and accuracy. The authors explored also the tradeoff between density and energy. In [12], the authors have identified the following QoS parameters tradeoffs between accuracy, delay, energy and density.

\section{QoS Mapping in Tree Based WSN}

\subsection{Problem Statement and Assumptions}

As mentioned earlier, QoS mapping is the process of translating QoS parameters from level to level, which is done by finding the relationship between the different QoS parameters, on the different levels of a distributed system. Typically, an entity in a distributed system has a layered architecture (fig. 1), which includes the user layer at the top, the network layer at the bottom and the application and system layers in the middle.

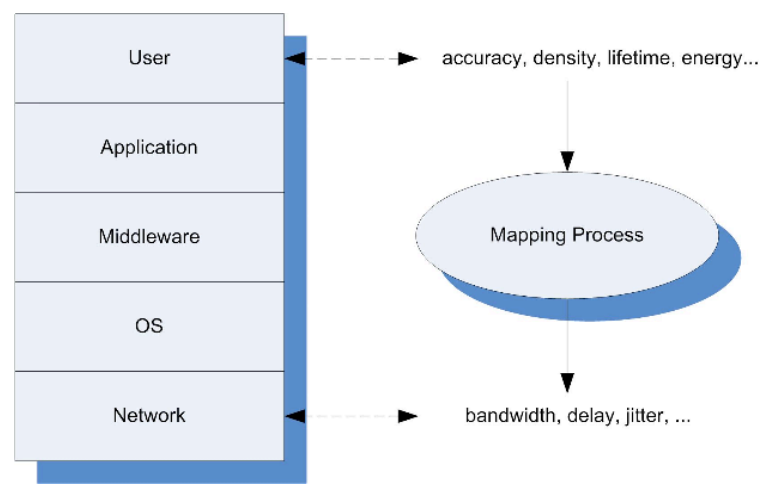

Fig. 1 Layered Architecture in Distributed Systems

On each layer, we could have different QoS requirements, depending on the entities on this layer. Starting from the top of the stack, we have the User and the Application entities with their QoS requirements as: 
1. Accuracy: It could be seen as the difference between a set of values assumed as the real values, and the value sensed by the network. Clearly, the number of active nodes in the network has a big impact on this amount of error: when the number of active nodes is increased, the computed error decreases.

2. Density: is the number of active nodes in the network divided by the volume. As mentioned above, network density has a direct impact on accuracy.

3. Energy: energy consumption is one of the most vital issues in WSN. Due to their small size, sensors suffer from their scarce resources like energy or memory. Thus, energy is usually an important QoS requirement for WSN users.

4. Lifetime: network lifetime could be seen as the duration for which a desired QoS is maintained. It could be also seen as the time until the first node (or a subset of nodes) fails, or runs out of energy.

When communication network is of concern, other QoS parameters are distinguished like bandwidth, delay, jitter, etc.

In order to guarantee a User level QoS, e.g. a certain amount of accuracy, we may have to add (or wake up) a number of nodes, thus we should try to answer the following questions: what does this amount of accuracy equals in terms of network density? And what does this amount of density equals in terms of lower level parameters (network level parameters) so that we can do the suitable reservations on that level?

Thus there is need to translate User level QoS, to Network level QoS (e.g. the bandwidth allocated for each node).

In the following, we discuss how user QoS requirements like accuracy and density in WSN, could be mapped into network QoS parameters like bandwidth. It has been noticed that in order to change the amount of accuracy (say increasing accuracy), we should add more nodes to the network (e.g. by turning them on), thus changing the network density. Once density is changed, the amount of bandwidth reserved for each node will change too.

In order to better understand the relationship between the density of a network and the bandwidth reserved for each node, we took as a model for our case of study, a tree-based clustered WSN, with a TDMA-based MAC protocol. Treebased clustered architectures are being widely used in WSN [13] because they are easily scalable and because of their unidirectional flow model, from leaves (sensor nodes) to the root (sink node).

We proceed to an example to better understand how we calculate the length of our TDMA superframe and how the changes in density affect directly the bandwidth reserved for each node. In this example we have a 5-levels tree-based clustered WSN (fig. 2).

Before we start we have to make some assumptions. First of all, nodes which are in the same cluster are considered in the same neighborhood, geographically speaking. Without loss of generality, we assume that initially, all level $h$ clusters in our network have the same number of nodes. This is justified because we want 
to start our scenario by having a balanced network; nevertheless, our network could evolve by adding or removing nodes from clusters, thus having eventually an unequal number of nodes in level $h$ clusters. Leaf nodes (on level 4 in this example) sense data, and forward it to their parent nodes in each cluster, which in turn forward it to their parent nodes, until the data arrives to the sink (level 0). Intermediate nodes could also be sensors, which means they could perform sensing too. In our case study, we take into account this fact and we compute the amount of time slots needed in the superframe for the intermediate nodes to perform their sensing activity. One could consider the simpler case where intermediate nodes are only relaying data, by simply omitting the part where those flows are computed, so whether intermediate nodes sense data or simply relay data is a choice made at the network implementation.

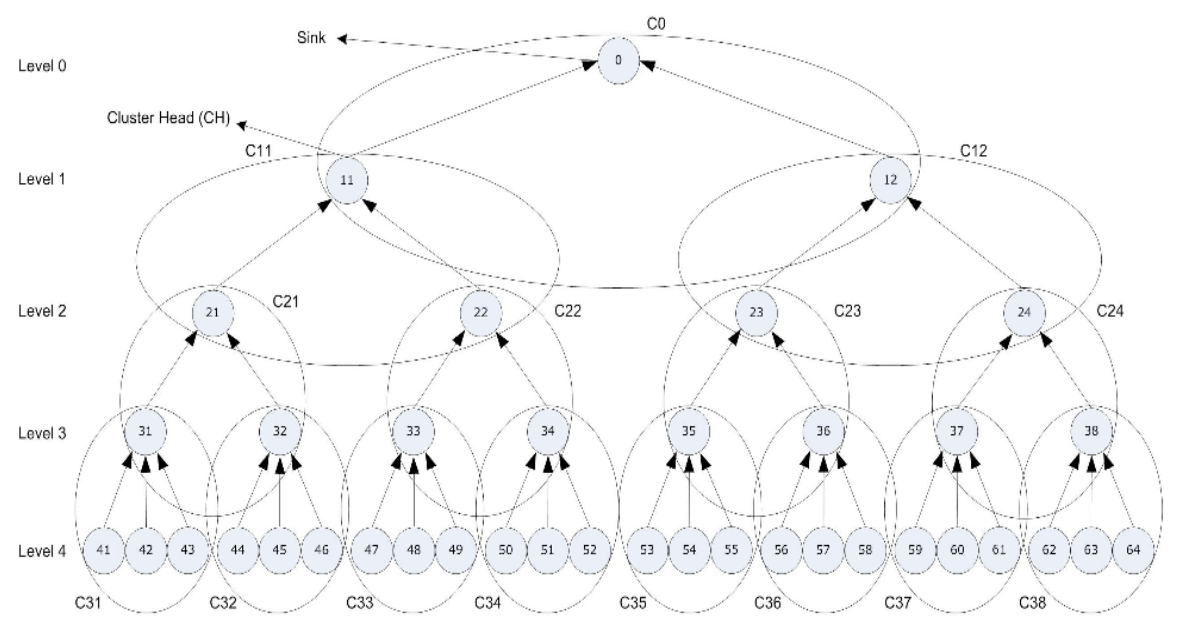

Fig. 2 Tree based clustered WSN

\subsection{Bandwidth Allocation}

Back to our example, nodes 21 and 22 are in the same cluster, so they are in the same neighborhood. Nodes 21 and 23 aren't in the same cluster, i.e. they cannot hear each other. For each cluster, there is a Cluster Head $(\mathrm{CH})$, which relays data received from its child nodes to its parent node and it could also perform data aggregation if there was data aggregation algorithms implemented on it. Data aggregation is out of the scope of this paper, so it's not considered in our example.

In order to compute the bandwidth available for each node, we have to compute the length of the TDMA superframe in this network first. The superframe must take into account all the flows, from leaf nodes to the sink, because intermediate nodes may share the same clusters, thus sharing the same neighbors. So the problem of overhearing (which often leads to collisions), must be taken into consideration when defining the superframe. 
We will proceed with the simplest case, and increase the problem complexity until we reach the most general case.

\section{i) First case: no simultaneous transmissions between clusters, sensing done by leaf nodes only.}

First, we consider the case of a WSN where only leaf nodes do sensing, therefore all the intermediate nodes' sensing flows are ignored. All simultaneous transmissions between same level clusters and "3 hops away nodes" are also ignored in this case.

The length of the TDMA superframe in a tree based clustered network with a depth equal to $H$, is obtained by the means of the following formula:

$$
\text { Length }_{H}=\left(\prod_{h=1}^{H} n_{h}\right) \times H
$$

where $H$ denotes the depth of the tree and $n_{h}$ denotes the number of child nodes of a $\mathrm{CH}$ on level $h-1$. This formula would be obvious if we observe the structure of the superframe (fig. 3). As we notice, the superframe is composed of 4 equal parts: 24 time slots for transmitting 24 flows from leaf nodes, 24 time slots for level 3 nodes to transmit the incoming flows from leaf nodes, 24 time slots for level 2 nodes to transmit incoming flows from level 3 and 24 time slots for level 1 nodes to transmit incoming flows from level 2 nodes.

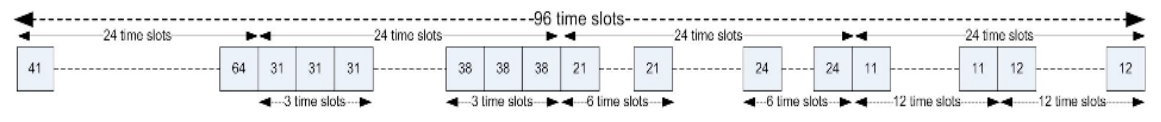

Fig. 3 TDMA superframe (first case)

ii) Second case: simultaneous transmissions by same level clusters, sensing done by leaf nodes only.

Now let us consider the case with simultaneous transmissions knowing that clusters of the same level can transmit their respective flows on the same time slots due to the fact that no overhearing could occur between separate clusters. Formula (1) is thus reduced to:

$$
\text { Length }_{H}=\sum_{k=1}^{H}\left(\prod_{h=\hbar}^{H} n_{h}\right)
$$

This is depicted in (fig. 4). As we notice, flows from clusters C31 to C38 are sent on the first, second and third time slots of the superframe because no overhearing could occur between those clusters. When the same rule is applied for each of the levels, we obtain formula (2). 


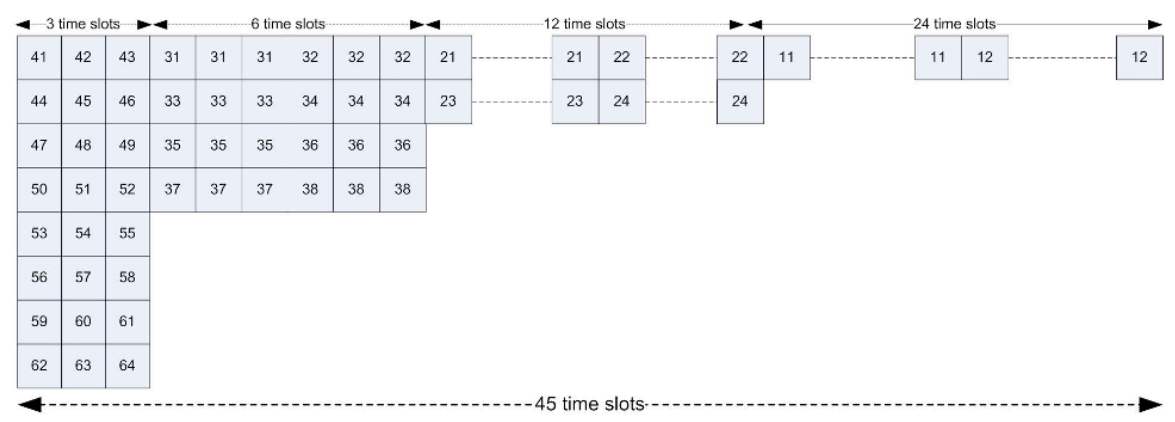

Fig. 4 Reduced TDMA superframe due to simultaneous transmissions (second case)

iii) Third case: simultaneous transmissions by same level clusters and 3 hops away nodes, sensing done by leaf nodes only.

Moreover, further optimization in the superframe length could be applied when taking into account the simultaneous transmissions from 3 hops away nodes. In fact, we noticed that nodes that are 3 hops away (e.g. node 41 and node 22, node 31 and node 12), could also transmit simultaneously without interference. In that case level 4 nodes could transmit their data along with level 2 nodes, and level 3 nodes could transmit their data with level 1 nodes (fig. 5). Clearly, the " 3 hop away nodes" optimization couldn't be applied on level 2 and level 1.

This rule is obviously applicable on any tree, regardless of its depth $H$. We could always transmit level $h$ flows with level $h-2$ flows simultaneously without interference. The formula would thus be the following:

$$
\text { Length } h_{H}=\prod_{h=1}^{H} n_{h}+\prod_{h=2}^{H} n_{h}
$$

This formula is the reduced form of (2). As we can see, we omitted the part concerning the flows from level 3 to level $H$, because they are transmitted simultaneously with each other as explained above.

\section{iv) Fourth case: simultaneous transmissions by same level clusters and 3 hops away nodes, sensing done by leaf nodes and intermediate nodes.}

In all of the above cases, intermediate nodes are only relaying data; they don't produce any flows of their own. Now we will consider the most complete case where intermediate nodes are also sensing and transmitting data. In that case, we should add to our formula the part in which we computed the number of time slots for the intermediate nodes to send their own flows. In fact, the number of time slots initially reserved in the superframe for level $h$ nodes should be increased by 


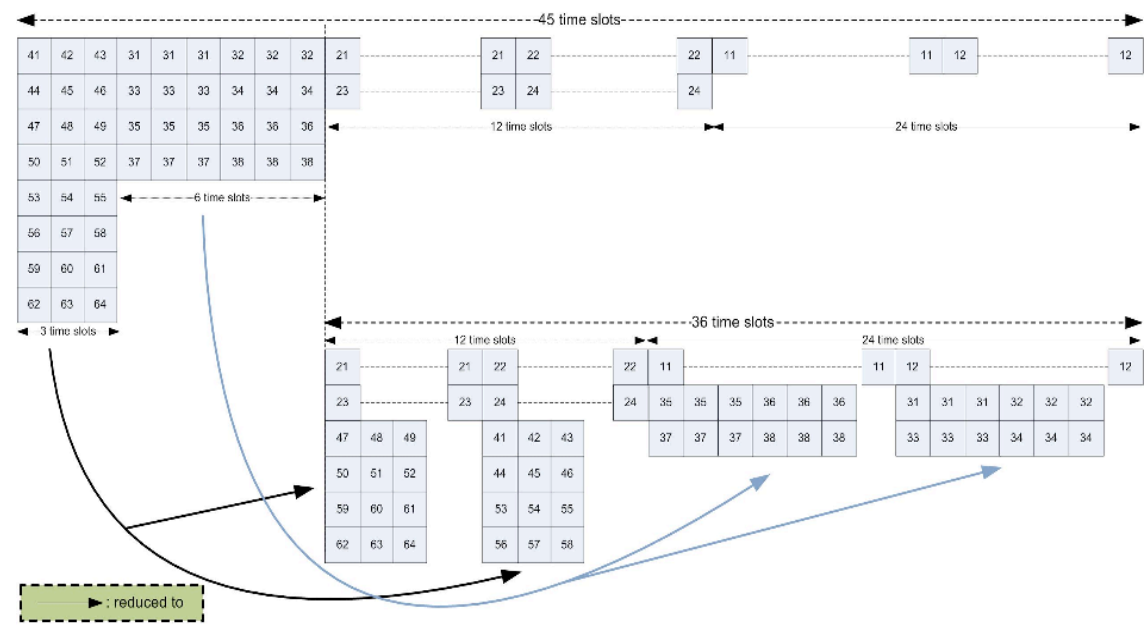

Fig. 5 Optimized TDMA superframe with simultaneous transmissions for 3 hops away nodes (third case)

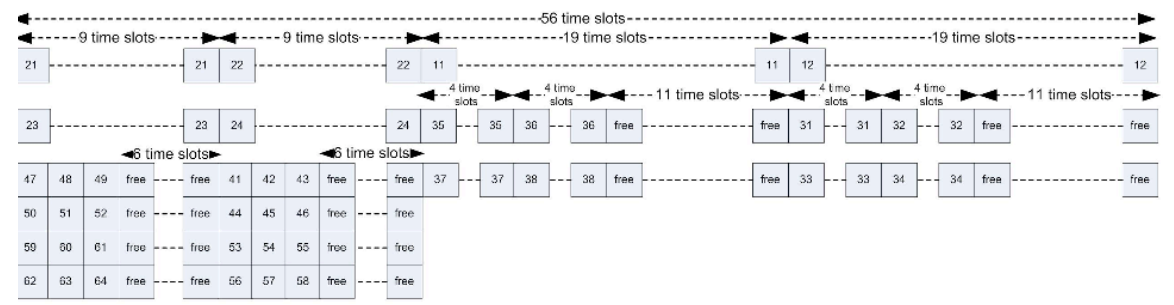

Fig. 6 TDMA superframe in the complete case (fourth case)

$F_{h}$, which corresponds to the number of child intermediate nodes' flows added to level $h$ nodes' own flows:

$$
F_{h}=\sum_{k=h}^{H-1}\left(\prod_{i=h}^{k} n_{i}\right)
$$

In our example, the number of slots we should add to level 2 nodes (nodes 21 and 22) is: $F_{2}=\sum_{k=2}^{H-1}\left(\prod_{i=2}^{k} n_{i}\right)=\mathrm{n}_{2}+\mathrm{n}_{2} \times \mathrm{n}_{3}=2+2 \times 2=6$ additional slots $(3$ for each node). This is depicted in (fig. 6), where there are 9 slots reserved for node 21 instead of 6 slots in the previous case. In order to compute the total number of slots added we should do the sum of $F_{h}$ for every level defining the superframe length, that is level 1 and level 2. So, the total number of slots added is: 


$$
F_{\text {total }}=\sum_{h=1}^{2} F_{h}=F_{1}+F_{2}=\sum_{k=1}^{H-1}\left(\prod_{i=1}^{k} n_{i}\right)+\sum_{k=2}^{H-1}\left(\prod_{i=2}^{k} n_{i}\right)=\left(n_{1}+n_{1} \times n_{2}+n_{1} \times n_{2} \times n_{3}\right)+\left(n_{2}+n_{2} \times n_{3}\right)=20
$$

Hereby, using formulas (3) and (4), the length of the superframe is computed as follows:

$$
\text { Length }_{H}=\prod_{h=1}^{H} n_{h}+\prod_{h=2}^{H} n_{h}+\sum_{k=1}^{H-1}\left(\prod_{i=1}^{k} n_{i}\right)+\sum_{k=2}^{H-1}\left(\prod_{i=2}^{k} n_{i}\right)
$$

The length of the superframe in the most general form is thus:

$$
\text { Length }_{H}=\prod_{h=1}^{H} n_{h}+\prod_{h=2}^{H} n_{h}+\sum_{k=1}^{H-1}\left(\prod_{i=1}^{k} n_{i} b_{i}\right)+\sum_{k=2}^{H-1}\left(\prod_{i=2}^{k} n_{i} b_{i}\right)
$$

where $b_{i}$ is a Boolean which equals 1 if level $i$ nodes are sensing data and $O$ if else. Now that we have the superframe length, we can compute the bandwidth ratio allocated for any level $h$ node. From formula (2) and (4) we obtain:

$$
B_{h}=\frac{\left(\prod_{k=h}^{H} n_{k}+\sum_{k=h}^{H-1}\left(\prod_{i=h}^{k} n_{i} b_{i}\right)\right)}{\operatorname{Length}_{H} \times n_{h}}
$$

Obviously, if we want to add leaf nodes to the network, we have to reschedule the superframe by adding $H$ slots for each added node. But, due to the fact that the superframe contains free time slots (fig. 6), we could add (a certain amount of) nodes to the network while just adding 2 additional slots (to the superframe) for each node added (the rest of the flows fit in the free slots). In order to compute the maximum number of leaf nodes, $N_{H}$, while preserving this rule, we have to calculate the number of time slots available in level $H$ starting from the number of time slots in level $l$ (using formulas 2 and 4 ). This is computed as follows:

$$
N_{H}=\left\{\begin{array}{cl}
v & \text { if } H=3 \\
\left\lfloor\left(\left\lfloor\left(v-n_{3}\right) / n_{3}\right\rfloor \ldots-n_{H-1}\right) / n_{H-1}\right\rfloor & \text { if } H>3
\end{array} \quad / v=\prod_{h=2}^{H} n_{h}+\sum_{k=2}^{H-1}\left(\prod_{i=2}^{k} n_{i} b_{i}\right)+b_{1}\right.
$$

In our example, all intermediate nodes are performing data sensing, so $b_{i}=1 \forall i \in[1 . . H] . N_{4}$ is computed as follows: 
$N_{4}=\left(\left(\prod_{h=2}^{4} n_{h}+\sum_{k=2}^{3}\left(\prod_{i=2}^{k} n_{i} b_{i}\right)+1-n_{3}\right) / n_{3}\right)=((2 \times 2 \times 3)+(2 \times 1+2 \times 1 \times 2 \times 1)$

$+1 \times 1-2) / 2)=8$ nodes. This means, we can add up to 8 leaf nodes in every cluster, while adding just 2 slots in the superframe for each added node. Beyond this number $\left(N_{H}\right)$, for every node added to the network we will have to add $H$ slots in the superframe. All of this is summarized in rule 9.

$$
B_{h}= \begin{cases}\frac{\left(\prod_{k=h}^{H} n_{k}+\sum_{k=h}^{H-1}\left(\prod_{i=h}^{k} n_{i}\right)\right)}{\operatorname{Length}_{H} \times n_{h}} & \text { if } n_{H}=n_{H \text { init }} \\ \frac{\left(\prod_{k=h}^{H} n_{k}+\sum_{k=h}^{H-1}\left(\prod_{i=h}^{k} n_{i}\right)\right)}{\left(\text { Length }_{H}+2\right) \times n_{h}} & \text { if } n_{H \text { init }}<n_{H}<=N_{H} \\ \frac{\left(\prod_{k=h}^{H} n_{k}+\sum_{k=h}^{H-1}\left(\prod_{i=h}^{k} n_{i}\right)\right)}{\left(\text { Length }_{H}+H\right) \times n_{h}} & \text { if } n_{H}>N_{H}\end{cases}
$$

\section{Simulations and Analysis}

In order to prove our formulas concerning the mapping between the different QoS parameters, we have conducted several simulations using the Network Simulator 2 (NS-2), with the following settings:

Table 1 Simulations settings

\begin{tabular}{ll}
\hline Simulation area & $1000 \times 1000 \mathrm{~m} !$ \\
\hline Time of simulation & $150 \mathrm{~seconds}$ \\
MAC Layer & TDMA \\
Number of nodes & 56 nodes (fig. 6) \\
Channel bit rate & $2 \mathrm{Mbps}$ \\
Data dissemination interval & $0.35 \mathrm{sec}$ \\
Data sensing interval & $0.35 \mathrm{sec}$ \\
Transmission range & $50 \mathrm{~m}$ \\
\hline
\end{tabular}


Below we discuss the main generic forms of relationships between QoS parameters backed up by simulations.

\subsection{Relationship Between Network Density and Bandwidth}

The relationship between those two parameters is explained in the previous section, where we proved that adding nodes to the network will decrease the bandwidth reserved for each node by an amount computed using rule (9). In rule (9), we presented three different formulas corresponding to three different cases, based on the number of leaf nodes in each $H$ cluster. Practically, it means that adding one more node to the network, will slightly increase the superframe length (i.e. slightly decrease the reporting frequency) thus reducing reserved bandwidth for each node. On one hand we have gained accuracy by increasing network density, and on the other we lost some accuracy while decreasing data freshness. A good tradeoff between those values would be having $N_{H}$ leaf nodes in each cluster, using all the free slots in the superframe and thus increasing accuracy, while slightly decreasing data freshness and bandwidth $\left(2^{\text {nd }}\right.$ case in rule 9$)$. Beyond this number, reporting frequency will highly decrease and thus affecting the data freshness parameter; beside, the bandwidth for each node will also decrease leading for example to the malfunctioning of the network (delay, jitter...).

In fig. 7, we report our simulation result using the example from the previous section, where level 4 clusters contain initially 3 sensors each. Sensors are added on a level 4 cluster, until we reach 12 sensors. We could notice how bandwidth decreases while adding nodes, and how it starts decreasing faster when it exceeds $N_{H}\left(N_{H}\right.$ is computed using formula 8$)$. These results validate our theoretical formulas presented in the previous section (formulas 6, 7, 8 and 9).

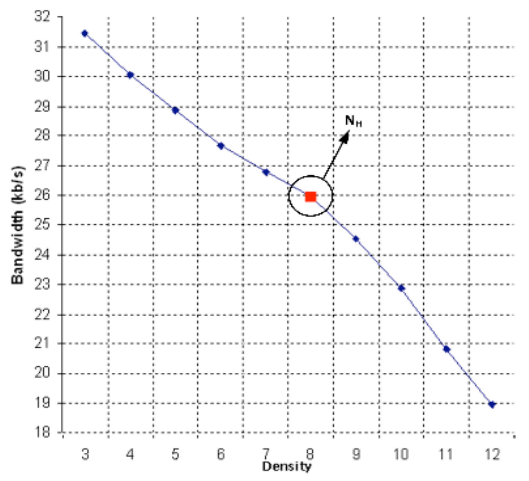

Fig. 7 Relationship between Network Density and Bandwidth

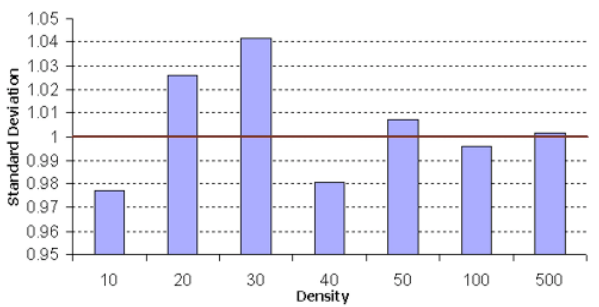

Fig. 8 Relationship between Accuracy and Density 


\subsection{Relationship Between Accuracy and Network Density}

Accuracy could be considered as the difference between the set of the real values and the set of the sensed values. This difference could occur because of the physical nature of sensors, or their distance to the target. This could be represented by the standard deviation, computed as follows:

$$
\text { Deviation }=\sqrt{\frac{1}{N-1} \times \sum_{i=1}^{N}\left(x_{i}-\bar{x}\right)^{2}}
$$

where $x_{i}$ is the sensed value, $\bar{x}$ is average value, and $N$ is the number of sensed samples. Obviously, we could minimize this amount of deviation by increasing the network density, i.e. by increasing the number of nodes collecting data, a technique called data redundancy. That could be done either by adding nodes to the cluster or by awaking sleeping nodes.

In our application (Temperature Sensing), we have varied the number of nodes sensing temperature, in order to analyze the relationship between the measured accuracy and the number of nodes sensing a certain phenomenon. Our sensed values follow a Gaussian distribution, with an average temperature of $25^{\circ} \mathrm{C}$, and a standard deviation of $1{ }^{\circ} \mathrm{C}$.

In fig. 9, we show how the standard deviation of the data samples converges toward the theoretical deviation (of $1^{\circ} \mathrm{C}$ ) as the network density increases.

The small amount of accuracy gained while moving from 100 sensors to 500 sensors for example, may raise the tradeoff issue between the gained accuracy and the cost incurred.

\subsection{Relationship Between Reporting Period, Network Density and Packet Size}

Reporting period or data freshness is in fact considered as another facet of accuracy. A fresher data (i.e. a shorter reporting period), corresponds to more accurate results. Packet size also affects accuracy: a bigger packet carries more accurate measurements.

Reporting period is directly related to the number of reporting nodes and it depends also on the packet size. In this case, modifying one parameter could affect the others. For example, in order to increase data freshness (i.e. decrease reporting period), we will have to decrease the superframe length, by either removing time slots (i.e. decreasing the number of nodes), or by decreasing the packet size al- 
lowed for each node. In both cases, accuracy will be affected negatively. So there is clearly a tradeoff to be done between those parameters.

Fig. 10 shows how reporting period increases when the network density increases, due to longer superframes. We could also notice that nodes allowed to send bigger packets (e.g. 1500 bytes) in their time slots are the most affected by the increase of density. In a WSN with 100 nodes deployed, allowing each to send 1500 bytes in its time slot, the reporting period reaches 0.6 seconds, while it is much shorter ( 0.04 seconds) when nodes are allowed to send 100 bytes in their time slots. In the first case, data freshness is affected while more accurate results are sent, while in the second case, data is sent more frequently but it's less accurate.

Understanding those relationships or mapping rules, will help us better evaluate the tradeoffs between the different QoS parameters, guaranteeing though a better QoS level for applications by fine tuning those parameters.

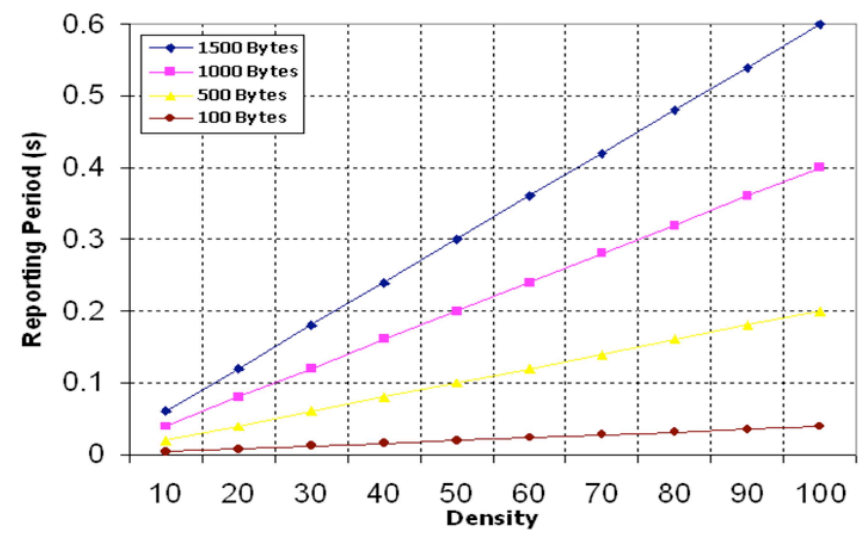

Fig. 9 Relationship between Density, Reporting period and Packet Size

\section{Conclusion}

QoS Support is one of the least explored tracks in WSN domain, although it has a crucial importance in many kinds of applications (e.g. real-time applications). In this paper we highlight an important issue in QoS Support: the QoS mapping. We discuss an approach for mapping user level QoS parameters (density and accuracy) to a network level QoS parameter (bandwidth). This was achieved by computing the TDMA superframe length in different scenarios, and presenting the bandwidth as a function of the superframe length and the network density.

We conduct also several simulations while giving the relationships between the different QoS parameters. Our experiments discuss relationships between density 
and bandwidth, accuracy and density, and between data freshness, density and packet size. One possible track we will try to explore in the future is to find the relationships between other QoS parameters like between density and delay for example. We will try also to investigate other kinds of MAC protocols like 802.11e.

\section{References}

1. S. Datta and T. Woody, Business 2.0 Magazine, February 2007 issue.

2. Technology Review Magazine (MIT), February 2003 issue.

3. G.J. Pottie and W.J. Kaiser: Wireless integrated network sensors. Communications of the ACM (2000).

4. W. Masri and Z. Mammeri: Middleware for Wireless Sensor Networks: A Comparative Analysis. In: Proceedings of the 2007 IFIP International Conference on Network and Parallel Computing - workshops (NPC 2007), China (September 2007).

5. R. Iyer and L. Kleinrock: QoS Control for Sensor Networks. In: Proceedings of ICC 2003, Alaska (May 2003)

6. J. Zhou and C. Mu: Density Domination of QoS Control with Localized Information in Wireless Sensor Networks. In: Proceedings of the 6th International Conference on ITS Telecommunications, China (June 2006)

7. J. Frolik: QoS Control for Random Access Wireless Sensor Networks. In: Proceedings of the IEEE Wireless Communications and Networking Conference, USA (March 2004)

8. B. H. Liu, P. Ray, S. Jha: Mapping Distributed Application SLA to Network QoS Parameters. In: Proceedings of the 10th International Conference on Telecommunications, Tahiti (February 2003)

9. Z. Mammeri: Framework for Parameter Mapping to Provide End-to-End QoS Guarantees in IntServ/DiffServ Architectures. In: Computer Communications, Volume 28, (June 2005)

10. M. Al-Kuwaiti, N. Kyriakopoulos, and S. Hussein: QoS Mapping: A Framework Model for Mapping Network Loss to Application Loss. In: Proceedings of the 2007 IEEE International Conference on Signal Processing and Communications, UAE (November 2007)

11. S. Tilak, N. B. Abu-Ghazaleh, W. Heinzelman: Infrastructure Tradeoffs for Sensor Networks. In: Proceedings of the 1st ACM International Workshop on Wireless Sensor Networks and Applications, USA (September 2002)

12. S. Adlakha, S. Ganeriwal, C. Schurgers, M. Srivastava: Poster Abstract: Density, Accuracy, Delay and Lifetime Tradeoffs in Wireless Sensor Networks - A Multidimensional Design Perspective. In: Proceedings of the ACM SenSys, USA (November 2003)

13. A. Koubaa, M. Alves, E. Tovar: Modeling and Worst-Case Dimensioning of Cluster-Tree Wireless Sensor Networks. In: Proceedings of the 27th IEEE International Real-Time Systems Symposium, Brazil (December 2006). 